\title{
La sostenibilidad como referencia para la gestión de los destinos turísticos
}

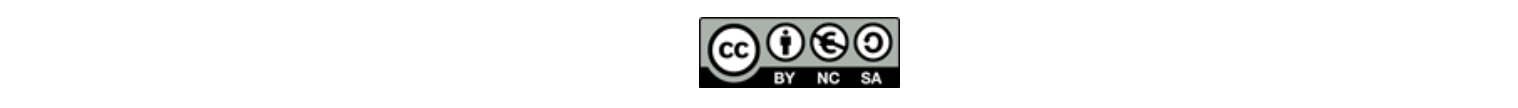

Sustainability as a reference for the management of tourist destinations.

Msc. Martha Omara Robert Beatón. ${ }^{1}$

Recibido: 17-12-2017 / Revisado: 18-12-2017 /Aceptado: 24-12-2017 / Publicado: 04-01-2018

Resumen. $\quad$ DOI: https://doi.org/10.33262/concienciadigital.v1i1.852

El presente trabajo tiene como objetivo determinar las herramientas, métodos y/o modelos que permitan evaluar la sostenibilidad en un destino turístico, tomando como base las que refiere la literatura científica consultada y a partir de parámetros definidos en investigaciones precedentes sobre el estudio de la sostenibilidad. Utilizando fundamentalmente los métodos de síntesis de la bibliografía sobre el tema objeto de estudio y el análisis comparativo. Los resultados de este análisis demostraron que las herramientas modelos y métodos no permiten evaluar la sostenibilidad desde un enfoque interdimensional, intradimensional y multidimensional que integre las dinámicas complejas que concurren en la gestión de un destino turístico, sentando las bases para realizar investigaciones desde otras áreas del conocimiento en la búsqueda de instrumentos dirigidos a reconocer y a comprender la visión holística de la sostenibilidad en los destinos turísticos.

Palabras claves: sostenibilidad, evaluación sostenibilidad, destinos turísticos.

\section{Abstract.}

The present work has as objective to determine the tools, methods and / or models that allow to evaluate the sustainability in a tourist destination, taking as a base those referred by the scientific literature consulted and from parameters defined in previous research on the study of sustainability. Using fundamentally the synthesis methods of the bibliography on the subject matter of the study and the comparative analysis. The

\footnotetext{
${ }^{1}$ Universidad de La Habana. Cuba. morobert@gmail.com
} 
results of this analysis showed that the models and methods tools do not allow to evaluate the sustainability from an interdimentional, intradimentional and multidimensional approach that integrates the complex dynamics that concur in the management of a tourist destination, setting the bases to carry out investigations from other areas of the knowledge in the search for instruments aimed at recognizing and understanding the holistic vision of sustainability in tourist destinations.

Keywords: sustainability, sustainability evaluation, tourist destinations.

\section{Introducción.}

Después de la conferencia de Río de 1992, se debate de qué forma el turismo debe desempeñar un papel dentro del de desarrollo sostenible. En 1995 se elabora la Carta de Lanzarote, en el marco de la Conferencia Mundial de Turismo Sostenible y en 1996 la Agenda 21 para la industria de viajes y turismo. En años posteriores se ha ido profundizando tanto en los diferentes ámbitos de la sostenibilidad aplicada el turismo como en los distintos niveles de actuación por los diferentes agentes. Alcanzando en el 2015 una agenda de desarrollo sostenible universal y transformadora, donde se ponen de manifiesto la experiencia de la comunidad internacional en materia de desarrollo de los últimos 20 años y se definen 17 objetivos (hasta el 2030) concretos y concisos en la búsqueda de un cambio de paradigma para las personas y el planeta; de ellos 5 responden específicamente a la actividad turística con el fin de mitigar las consecuencias de los modelos de desarrollo establecidos; declarando el 2017, “Año Internacional del Turismo Sostenible” [1].

Los estudios más recientes sobre sostenibilidad en el mundo han tratado la creación de herramientas para medir y/o evaluar varios aspectos de la sostenibilidad en el turismo [2;3;4]. La Organización Mundial del Turismo (OMT) $(1998,2005,2007)$ ha emitido varias guías para la evaluación del desarrollo sostenible en este sector. En el año 2005 esta organización emitió una guía práctica (lista de indicadores) para contribuir a erradicar problemas con respecto a la gestión del desarrollo sostenible en destinos turísticos. [5;6;7].

En Cuba la mayoría de los estudios sobre sostenibilidad turística de destinos han sido realizados a partir de la determinación y evaluación de indicadores divididos de acuerdo a las dimensiones de la sostenibilidad. Esto ha estado determinado por la elaboración de la propuesta de indicadores de sostenibilidad para el turismo en Cuba, documento elaborado de conjunto entre el Ministerio de Turismo (MINTUR) y el Ministerio de Ciencia Tecnología y 
Medio Ambiente (CITMA) cubanos en el año 2003, el cual, aunque todavía insuficiente, ha constituido pauta para el desarrollo de las investigaciones más recientes con respecto a este tema en el país.

Entre esas investigaciones se encuentran las realizadas por Blanco (2016) para el destino Cuba, Delis y Echarri (2015) en el Centro Histórico de La Habana, sobre varios destinos de naturaleza cubanos. Estos estudios, aunque han coincidido en el método o herramienta a utilizar, difieren, al igual que los estudios internacionales sobre este tema, en cuanto al enfoque aplicado, así como en el proceso de determinación de los indicadores y, por supuesto, en los indicadores determinados para cada caso. [8;9].

Ante la multiplicidad de enfoques, modelos y herramientas, así como las diferencias entre las investigaciones realizadas siguiendo los mismos métodos para evaluar la sostenibilidad del desarrollo de los destinos turísticos, se hace necesario establecer una base uniforme para definir niveles de sostenibilidad y al mismo tiempo permita establecer comparaciones espaciales y temporales entre diferentes destinos turísticos, por lo que se plantea la siguiente pregunta de investigación: ¿Qué herramienta, métodos y/o modelos permiten evaluar la sostenibilidad en un destino turístico?

De acuerdo al problema científico anterior se plantea entonces como objetivo de la presente investigación: Determinar herramienta, métodos y/o modelos que permitan evaluar la sostenibilidad en un destino turístico.

La investigación que se propone intenta una visión mucho más abarcadora que permita determinar las herramientas métodos y/o modelos que pueden ser utilizadas para evaluar la sostenibilidad de los destinos turísticos desde un enfoque interdimensional, intradimensional y multidimensional que integre las dinámicas complejas que en él se producen.

\section{Método}

La investigación es de tipo descriptiva, pues como su tipología lo indica en el desarrollo del estudio científico se describe lo más preciso posible el fenómeno que se investiga [10]. Se determina el comportamiento de las herramientas métodos y modelos para evaluar la 
sostenibilidad que reconoce la literatura científica consultada. El proceso metodológico se desarrolló en 3 etapas, las cuales se caracterizan a continuación:

Etapa preparatoria: el completamiento de las bases teóricas se basó en el análisis bibliográfico como método empírico, que permitió la identificación y consulta de fuentes de información necesarias que permitieron extraer y recopilar datos esenciales para la conformación de la investigación. Los principales tópicos de consulta desde la bibliografía especializada en los temas de sostenibilidad, y de gestión de los destinos. La utilización de este método permitió reconocer las prácticas más generalizadas de herramientas modelos y métodos para evaluar la sostenibilidad en destinos turísticos.

Etapa trabajo de mesa: como principal técnica de recopilación de información en esta etapa partió de una amplia revisión bibliográfica especializada sobre el tema como se refiere en la etapa anterior, que permitió realizar una comparación de herramientas modelos y métodos que reconoce la literatura científica como los más utilizados, siguiendo los parámetros de comparación declarados por Torres (2016): Enfoque Integrado, determinación de equilibrio, establece relaciones complejas entre elementos o variable, compara resultado con objetivos de sostenibilidad, establece áreas de mejora, determina estado de sostenibilidad general y analiza la tendencia. Se analizó el comportamiento por cada parámetro desde un análisis matemático expresando en porcientos la cantidad de parámetros para cada herramienta, modelo y método.[11].

En un segundo momento de esta etapa se realiza un repaso, a los indicadores por ser la herramienta que ha demostrado una mayor utilización en la evaluación de la sostenibilidad de los destinos para ellos se tomaron las propuesta que reconoce la literatura científica tanto internacional y nacional como las más extendidas y generalizadas Asociación de Estados del Caribe (AEC) por su nombre en español (1999), el Ministerio de Turismo de Cuba(MINTUR)de conjunto con el Ministerio de Tecnología y Medio Ambiente(CITMA)(2003),la Organización Mundial del Turismo(OMT)(2005) la Unión Europea, (2016).[12;13;14;15].

Etapa final: se organizó y procesó la información obtenida, lo cual condujo al análisis de resultados, el cual responde al objetivo general planteado en la investigación enfocado sobre la base de determinar herramienta, modelo y método que permitan evaluar la sostenibilidad de un destino turístico. 


\section{Resultados}

Por la complejidad que implica la evaluación de la sostenibilidad en los destinos turístico se hace necesario un análisis de herramientas, modelos y métodos. Siguiendo los parámetros declarados por Torres (2016) [11], puede concluirse que:

- Solamente los sistemas de indicadores (herramientas) son los que permiten comparar los resultados obtenidos con los objetivos de la sostenibilidad no comportándose de la misma manera los (métodos) lo logran desde: el índices de sostenibilidad e indicadores compuestos y en el caso de los (modelos) exhiben un mejor comportamiento: análisis de la huella ecológica, AMOEBA, panel de la sostenibilidad, barómetro de la sostenibilidad.

- Las herramientas y métodos permiten realizar enfoque integrado, no así los modelos, sin embargo, de manera general (las herramientas, métodos y modelos) no determinan estado de equilibrio, ni estado de sostenibilidad general.

- Mayoritariamente (las herramientas, métodos y modelos) no permiten establecer relaciones complejas entre elementos o variables a excepción de razonamiento cualitativo (herramienta), análisis emergético (modelo) sistema-entorno y sistemas de cuentas medioambientales y económicas (métodos).

- De modo general no permiten análisis de tendencia sin embargo en todos los casos se establecen áreas de mejora, que permiten una retroalimentación en la búsqueda de un buen desempeño, de buenas prácticas en función de los objetivos del destino.

En relación con lo que se ha discutido acerca de las herramientas, modelos y métodos, a modo de resumen se muestra una tabla de su comportamiento por parámetros.

Tabla N 1 Comportamiento de los parámetros en función de las herramientas, métodos y modelos.

\begin{tabular}{lcccccccc}
\hline & $\begin{array}{c}\text { Enfoque } \\
\text { Integrado }\end{array}$ & $\begin{array}{c}\text { Determinació } \\
\text { n de } \\
\text { Equilibrio }\end{array}$ & $\begin{array}{c}\text { Establece } \\
\text { relaciones } \\
\text { complejas } \\
\text { entre } \\
\text { elementos } \\
\text { o variable }\end{array}$ & $\begin{array}{c}\text { Compara } \\
\text { resultado } \\
\text { con } \\
\text { objetivos } \\
\text { de } \\
\text { sostenibilid } \\
\text { ad }\end{array}$ & $\begin{array}{c}\text { Establece } \\
\text { áreas de } \\
\text { mejora }\end{array}$ & $\begin{array}{c}\text { Determina } \\
\text { estado de } \\
\text { sostenibilida } \\
\text { d general }\end{array}$ & $\begin{array}{c}\text { Analiza la } \\
\text { tendencia }\end{array}$ & Total \\
\hline Herramientas & $100 \%$ & $25 \%$ & $25 \%$ & $25 \%$ & $100 \%$ & $75 \%$ & $25 \%$ & 4 \\
\hline Métodos & $100 \%$ & $33,3 \%$ & $33,3 \%$ & $66,6 \%$ & $100 \%$ & $100 \%$ & $33,3 \%$ & 3 \\
\hline Modelos & $33,3 \%$ & $8,3 \%$ & $16,6 \%$ & $33,3 \%$ & $100 \%$ & $8,3 \%$ & $16,6 \%$ & 12 \\
\hline
\end{tabular}


Fuente: Elaboración propia

\section{Resumen del análisis comparado de las propuestas de indicadores}

Análisis de la propuesta de indicadores planteada por la Asociación de Estados del Caribe (AEC), 1999.

La Asociación de Estados del Caribe propone un conjunto de indicadores para armonizar los criterios de categorización para la inclusión de esos destinos en la Zona de Turismo Sostenible del Caribe, los cuales fueron identificados y concertados a través de los trabajos del Grupo de Expertos en Indicadores de Sostenibilidad del Comité Especial de Turismo Sostenible de la AEC.

La propuesta presenta 14 indicadores con una mayor concentración de los mismos en la dimensión ambiental (ocho), sociocultural (cuatro) y económica (dos).

Análisis de la propuesta de indicadores planteada por el Ministerio de Turismo de Cuba (MINTUR) en conjunto con el Ministerio de Ciencia, Tecnología y Medio Ambiente de Cuba (CITMA), 2003.

La propuesta de indicadores para el destino Cuba, la cual abarca todos los indicadores de sostenibilidad turística de la (AEC), los criterios de selección estuvieron basados en las características del área, así como los intereses de los gestores y los decisores (por lo que exceptúa el de prostitución infantil ya que no responde a las problemáticas de Cuba) e incorpora otros 16 que resultan de importancia para el logro de los objetivos y metas del desarrollo turístico.

Esta selección comprende un total de 29 indicadores, es muy general y poco específica para los destinos turísticos cubanos. En ellos, hay una mayor presencia de la dimensión ambiental (17 indicadores representando el $61 \%$ del total) con respecto a la económica (9 indicadores $22.5 \%$ ) y la sociocultural (8 indicadores $16.5 \%$ ).

Análisis de la propuesta planteada en la Guía Práctica de Indicadores de Desarrollo Sostenible para los Destinos Turísticos de la OMT (2005).

Esta propuesta ofrece elementos y referencias para elaborar indicadores que contribuyan a atenuar los problemas o dificultades en materia de política y gestión que puedan encontrarse 
en los diferentes destinos, por lo que no constituye una fórmula de indicadores, sino que está encaminada a generar ideas que promuevan propuestas.

En total se examinan 13 cuestiones generales que abarcan en su conjunto 42 comunes específicas, que a vez su vez responden a 231 componentes y a una propuesta de 750 indicadores. El análisis permitió conocer además que predominan las cuestiones e indicadores pertenecientes a la dimensión sociocultural por encima de la ambiental y la económica, destacándose como elemento notorio que la cuestión seis (Aprovechamiento de los beneficios económicos del turismo) es la que presenta el mayor número de cuestiones específicas (siete), de componentes (34) y de indicadores (153).

Esta guía presenta además una selección de las cuestiones básicas (12) e indicadores básicos (29) De las cuestiones básicas predominan las que responden a la dimensión ambiental con un total de seis, para 14 indicadores, lo que representa el 50\% del total.

\section{Propuesta de indicadores de sostenibilidad planteada por la Unión Europea, 2016.}

El Sistema de Indicadores del turismo europeo (ETIS), por sus siglas en inglés pretende ayudar a los destinos a monitorear y medir el desarrollo del turismo sostenible.

El mismo propone un total de 37 indicadores divididos en cuatro categorías: gestión del destino (tres indicadores), valor económico (nueve indicadores), impacto social y cultural (10 indicadores) e impacto ambiental (15 indicadores).

Para una mejor comprensión de los resultados se muestran los gráficos 1y2 que resumen el comportamiento general de las propuestas analizadas.

Gráfico N 1: Comportamiento por dimensiones por cada propuesta.

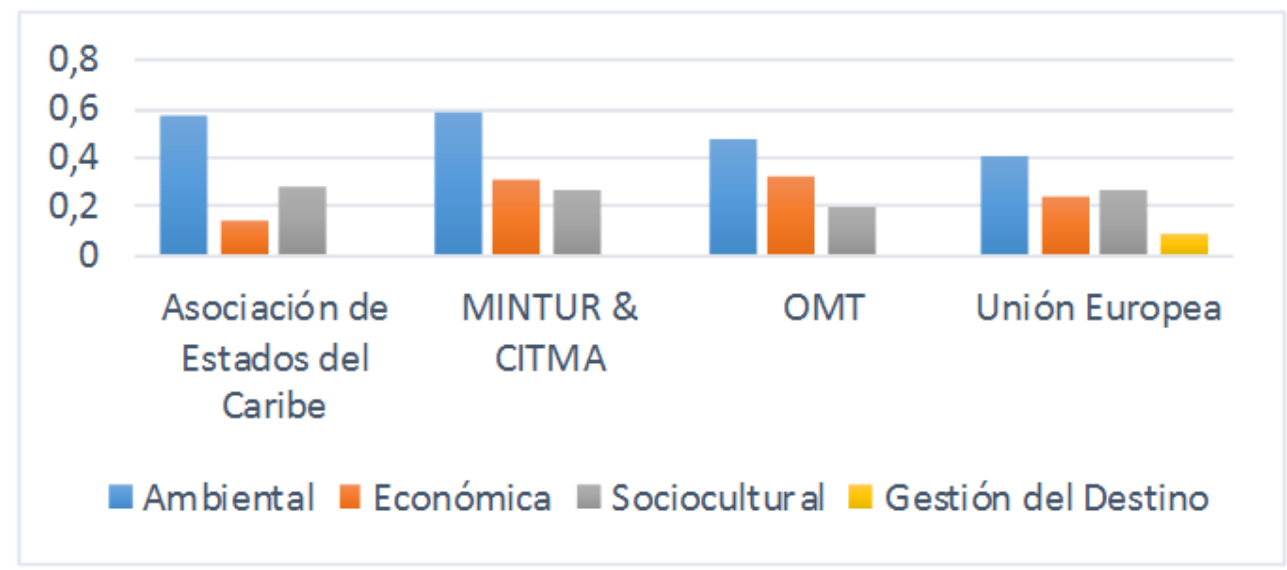

Fuente: Elaboración Propia. 
Gráfico N 2: Comparación porcentual entre dimensiones.

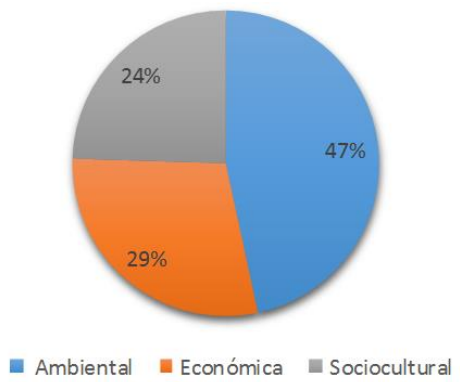

Fuente: Elaboración Propia.

Entre los últimos estudios que se realizan en Cuba en la búsqueda de herramientas para evaluar la sostenibilidad de los destinos hay que destacar los desarrollados desde la Facultad de Turismo de conjunto con el MINTUR la cual plantea como parte de sus resultados que los indicadores son la herramienta más utilizada en el presente y probablemente lo seguirán siendo en el futuro preferiblemente en combinación con otro instrumento, al mismo tiempo asevera que las propuestas nacen de fuentes gubernamentales y Blanco (2016) que se centra específicamente en lograr una propuesta de indicadores para evaluar la sostenibilidad del turismo del destino Cuba a partir de la disponibilidad de datos desde fuentes oficiales de acceso público. [8;11].

El estudio partió del análisis de la propuesta realizada por CITMA-MINTUR (2003) y la OMT (2005) contrastando las coincidencias de indicadores con la información que se dispone en la ONEI como única fuente gubernamental de acceso público en Cuba permitiendo arribar a una nueva propuesta de indicadores para evaluar el destino Cuba presentando las siguientes características [6;13]:

- La propuesta tiene un total de 22 indicadores, 11 perteneciendo a la dimensión económica, cuatro a la dimensión ambiental y siete a la social, reflejando un desequilibrio entre las dimensiones de la sostenibilidad.

- No existen una continuidad en las series de datos. 
- Cuando se refiere al nivel territorial que comprende el indicador y este es provincial o municipal, solamente se recogen los datos referidos a la provincia de La Habana y a sus municipios.

\section{Discusión}

De modo general las herramientas, métodos y modelos no responden al análisis de la sostenibilidad de un destino según los parámetros seleccionados, respondiendo solo al 100\% en el caso de establecer áreas de mejora, lo que denota las limitaciones de los mismos y los vacíos que quedan para evaluar la sostenibilidad de un destino, partiendo de que coexisten dos grandes complejidades el de llevar a la práctica el concepto de la sostenibilidad y el de la gestión de un destino con todos los procesos que en él se producen. Aunque es significativo este comportamiento positivo de todos en responder a establecer áreas de mejora si se tiene en cuenta que permiten una retroalimentación en la búsqueda de un buen desempeño y de aplicar buenas prácticas en función de los objetivos del destino.

Siguiendo una misma lógica de discusión la primera propuesta de indicadores (AEC) presenta un desequilibrio marcado entre las dimensiones, lo que limita la relación específica causa/efecto entre el turismo y las diferentes dimensiones de la sostenibilidad.

La propuesta de Cuba aunque más condensada denota la falta de equilibrio entre las dimensiones, no es capaz de medir el nivel de la sostenibilidad del turismo en los diferentes destinos del país y no permite una comparación entre destinos y en el tiempo, dificultando monitorear el comportamiento de la actividad turística determinado fundamentalmente por la falta de información.

La OMT por su parte desde su propuesta tiene un enfoque más abarcador en tanto asume un mayor número de cuestiones a evaluar en las diferentes dimensiones, facilitando su adecuación a diferentes tipos de espacios turísticos y recomendando un conjunto mínimo para realizar la descripción del estado de un destino, aunque no permite realizar análisis más complejos, si logra comparar comportamientos entre ellos; pero evidenciando desequilibrio a la hora de evaluar las relaciones inter e intradimensionales de la sostenibilidad en un destino turístico.

La Unión Europea limita el análisis holístico del destino con la selección de tres indicadores dentro de la categoría gestión del destino, ¿cabría preguntarse el resto no responden a la gestión del destino? Los mismos son seleccionados para ser parte integrante de los procesos 
de planificación, gestión y monitoreo, se debe partir del criterio de que entre ellos se producen relaciones intradimensionales tratándose de interacciones y de enfoques transectoriales, transdiciplinarios y transversales.

La propuesta más reciente de indicadores para el destino Cuba, aun cuando su comportamiento difiere del que evidenciaron el resto de las propuesta analizadas, donde la dimensión ambiental era la que ocupaba el mayor número de indicadores, en este caso ocupado por la dimensión económica, de igual manera en general el comportamiento es el mismo por el desequilibrio entre las dimensiones que evalúan la sostenibilidad y la incapacidad de responder al enfoque que la misma exige.

Otros elementos que deben tenerse en cuenta para seleccionar indicadores es que la sostenibilidad del destino puede estar condicionada por la percepción de quienes construyen los indicadores lo que puede traer aparejada subjetividad, impactos que pueden quedar solapados, así como una visión unidimensional. La evaluación del desarrollo sostenible no puede significar solo cuidar ecosistemas y recursos, sino promover el desarrollo equilibrado de los componentes medioambientales, socioculturales y económicos.

\section{Conclusiones}

- Se pudo determinar de manera general que las prácticas más generalizadas (herramientas, modelos y métodos) para evaluar la sostenibilidad aun no logran el enfoque multidimensional de las dinámicas complejas que concurren en un destino, lo que evidencia la necesidad de realizar investigaciones en la búsqueda de nuevos instrumento desde otras áreas del conocimiento dirigidos a reconocer y a comprender la visión holística de la sostenibilidad en los destinos turísticos y que al mismo tiempo disminuyan el carácter subjetivo de las variables que integran las dimensiones de la sostenibilidad.

- Si bien el estudio demuestra que los indicadores son la herramienta más utilizada hasta hoy para evaluar la sostenibilidad de los destinos, el análisis comparativo de las propuestas reconoce las limitaciones que presentan, demostrando la falta de equilibrio entre las dimensiones, impidiendo determinar niveles de sostenibilidad en los destinos turísticos así como comparar comportamientos espaciales y temporales. 


\section{Referencias bibliográficas.}

ORGANIZACIÓN DE LAS NACIONES UNIDAS. Nuestro Futuro Común o Informe Brutland. 1987. Disponible en: http://www.tij.uia.mx/elbordo/vol05/. (consulta: octubre del 2017).

FILIMONAU, V., DICKINSON, J. and ROBBINS, D., The carbon impact of short-haul tourism: a case study of UK travel to Southern France using life cycle analysis. Journal of Cleaner Production, 2014. 64, pp.628-638

PÉREZ ALBERT, Y, NEL·LOENDREU, M., Propuesta de indicadores para evaluar la sostenibilidad de la actividad turística. El caso del Valle de Viñales (Cuba. Anales de Geografía de la Universidad Complutense, 2013. 33(1), pp.193-210

BLANCAS, F. J. et al. (2010): "El indicador sintético DCP como instrumento de medición de la sostenibilidad turística" XVII Jornadas ASEPUMA - V Encuentro Internacional. Vol. Actas_17 Issue 1.

OMT: "Desarrollo Turístico Sostenible: Guía para Administraciones Locales" Madrid, España. 1998

OMT "Indicadores de desarrollo sostenible para los destinos turísticos. Guía práctica" Madrid. España. 2005.ISBN 92-844-0838-5

OMT ( “A Practical Guide to Tourism Destination Management” Madrid, España. 2007

BLANCO RÍOS, ADOLFO ARMANDO. Propuesta de indicadores para evaluar la sustentabilidad turística del destino Cuba. Trabajo de Diploma. Universidad de la Habana. Facultad de Turismo. La Habana. Cuba. 2016

ECHARRI Chávez, Maité, DELIS, C.D, “Evaluación de indicadores de sostenibilidad turística para el Centro Histórico de La Habana en el período 2010-2014" Tesis en opción al título de Máster en Gestión Turística. Facultad de Turismo, Universidad de La Habana. 2015.

PERELLÓ RODRÍGUEZ, José Luis. "La edición y publicación de los resultados de la investigación” Clase Metodológica Instructiva. Facultad de Turismo. Universidad de La Habana. 2014.

TORRES HECHAVARRIA, Lisandra. "Propuesta de esquema metodológico para la evaluación de la sostenibilidad del desarrollo turístico de destinos caso La Habana" Tesis Doctoral. Universidad de La Habana, Cuba. 2016.

ASOCIACIÓN DE ESTADOS DEL CARIBE. "Declaración para el establecimiento de la zona de turismo sustentable del Caribe, II Cumbre de Jefes de Estado y/o Gobierno de la A.E.C.”, Santo Domingo, 1999. 
CITMA - MINTUR. "Indicadores de sostenibilidad para el turismo en Cuba", Documento preliminar, La Habana, 2003.

ORGANIZACIÓN MUNDIAL DEL TURISMO (OMT). Indicadores de desarrollo sostenible para los destinos turísticos. Guía práctica. Madrid, España. 2005.

EUROPEAN COMMISSION. "The European Tourism Indicator System. ETIS toolkit for sustainable destination managment", 2016. Disponible en: http://ec.europa.eu/growth/sectors/tourism/offer/sustainable/indicators/index_en. htm. 
Para citar el artículo indexado.

Robert Beatón, M. (2018). La sostenibilidad como referencia para la gestión de los destinos turísticos. ConcienciaDigital,1(1), 34-46.

https://doi.org/10.33262/concienciadigital.v1i1.852

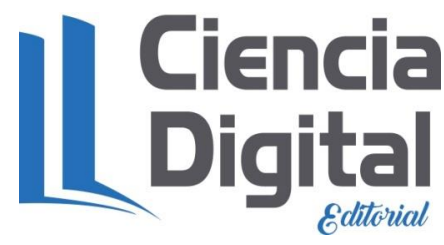

El artículo que se publica es de exclusiva responsabilidad de los autores y no necesariamente reflejan el pensamiento de la Revista Conciencia Digital.

El articulo queda en propiedad de la revista y, por tanto, su publicación parcial y/o total en otro medio tiene que ser autorizado por el director de la Revista Conciencia Digital.
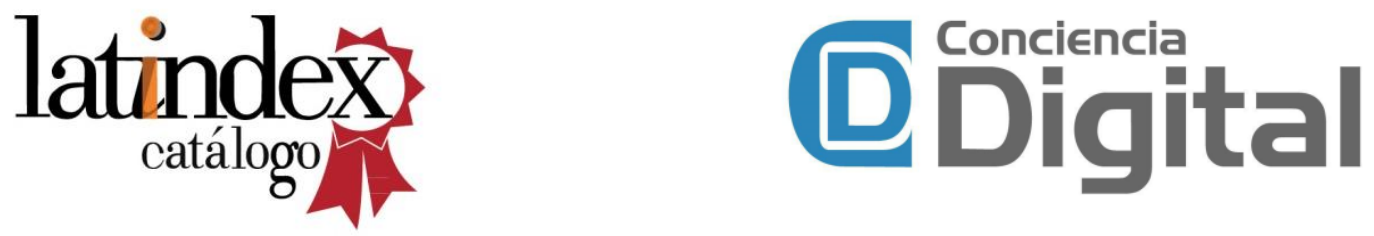